\title{
Electropolymerized Porous Polymer Films on Flexible Indium Tin Oxide Using Trifunctional Furan Substituted Benzene Conjugated Monomer for Biosensing
}

Supreetha Paleyanda Ponnappa, ${ }^{1}$ Jennifer MacLeod, ${ }^{1 \#}$ Muhammad Umer, ${ }^{2 \#}$ Narhsone Soda, ${ }^{3}$ Amandeep Singh Pannu, ${ }^{1}$ Muhammad J. A. Shiddiky, ${ }^{2,3}$ Godwin A.Ayoko, ${ }^{1}$ Anthony P. O’Mullane, ${ }^{1 *}$ Prashant Sonar $^{1 *}$

1. School of Chemistry, Physics and Mechanical Engineering, Queensland University of Technology (QUT), Brisbane, QLD 4001, Australia.

2. Queensland Micro- and Nanotechnology Centre (QMNC), Griffith University, Nathan Campus, QLD 4111, Australia

3. School of Environment and Science, Griffith University, Nathan Campus, QLD 4111, Australia.

\# Both authors contributed equally to this work

\begin{abstract}
In recent years, conducting polymers is playing a significant role in the field of display devices, transistors, solar cells, sensors and electrochromic windows due to their outstanding optoelectronic and semiconducting properties due to their conjugated backbone. One potential application that is not as widely explored using these materials is biosensing, where advantage is taken of the porosity that can be generated by polymerization of a three-dimensional network. There are various approaches for producing conjugated microporous polymers using trifunctional or multifunctional monomers synthesised via chemical or electrochemical methods. In this work, we have used electropolymerization to synthesize conjugated polymer films on a working electrode of Flexible Indium Tin Oxide (FITO) using a trifunctional conjugated monomer 1, 3, 5-tri(furan-2-yl) benzene (TFB). There are several parameters which influences the formation of a porous polymer film and the most critical ones are substrate conductivity, roughness, method of electropolymerization and choice of electrolyte. These porous electropolymerized films were characterised using UV-Vis spectroscopy (UV-Vis), Xray Photoelectron Spectroscopy (XPS), surface profilometry, four-point probe conductivity measurements, Scanning Electron Microscopy (SEM). The polymer films that were electropolymerized using chronoamperometry rather than repetitive potential cycling
\end{abstract}


demonstrated a more suitable morphology to trap DNA/RNA analytes for biosensing applications.

\section{Introduction}

In the last few decades, conducting polymers have gained significant attention from the organic electronics community due to their interesting optoelectronic and semiconducting properties arising from their conjugated backbone. ${ }^{1}$ These conjugated polymers can be used as an active thin film material in various electronic devices such as organic light emitting diodes (OLEDs), organic field effect transistors (OFETs), organic photovoltaics (OPVs), logic circuits as well as chemical and biochemical sensors. ${ }^{2}$ Among the various classes of conjugated polymers, conjugated microporous polymers (CMP's) are predominant classes of materials and were first reported by Cooper in 2007. ${ }^{3-4}$ CMP's are a class of conjugated polymer that vary slightly compared to regular conjugated polymers due to the $\pi$-conjugation in their backbone that are linked in the form of three-dimensional (3D) networks. This unique feature produces a porous conjugated skeleton network which makes them different to more conventional conjugated polymer systems that form compact thin films. ${ }^{4}$

Microporous polymer films have recently gained importance due to their porosity and therefore inherently larger surface area. The formation of a microporous conjugated backbone with various functional groups attached to the backbone has resulted in many applications beyond organic electronic devices like energy storage,${ }^{5}$ gas capturing molecules, ${ }^{6-8}$ drug delivery, ${ }^{9-10}$ catalysis ${ }^{11-13}$ and sensors. ${ }^{14-16}$ In general, conjugated monomers that contain rigid backbone planar molecules without long alkyl chains are difficult to solubilise, which is why designing monomers in a non-planar fashion requires attaching functional groups such as furan, thiophene, melanoic acid, carboxylic acid and solubilising groups (such as alkyl chains) to enhance their solubility in common organic solvents. The Monomer solubility is essential for the formation of good quality polymer films using the electropolymerization technique.

Conventional chemical techniques for polymer synthesis such as Suzuki-Miyura coupling, ${ }^{17}$ Stille coupling, ${ }^{18}$ Sono-Gashira-Hagihara cross-coupling, ${ }^{19-20}$ Yamamoto coupling, ${ }^{21-22}$ oxidative coupling, ${ }^{23}$ heat treatment ${ }^{24}$ or direct arylation ${ }^{25}$ can have some drawbacks. The common ones are these techniques needs high-cost catalysts such as nickel or palladium in combination along with ligands such as triphenyl phosphine, dibenzylideneacetone and bis(cyclooctadiene). During the chemical polymerization, a huge amount of polymer can be synthesized for various applications, however, batch to batch 
molecular weight variation can affect optoelectronic properties. Another critical challenge is the synthesis of monomers which require suitable chemical functional groups such as di-halo, bis-boronic, bis-stannyl, bis-acetylene for successful polymerization which adds more synthesis steps making the production of these types of monomers time consuming and expensive. In order to resolve these issues, an alternative method known as electropolymerziation can be used where the polymer film can be deposited directly on a conducting substrate if a suitable oxididation site is available on the electron rich (donor) monomer. In the case of electropolymerization, the film thickness and the dimensions of the cross-linked polymer network can be tuned by changing the concentration of the monomer and the applied potential or sweep rate during potential cycling deposition ${ }^{26}$ which also influence the homogenity of the polymer film. ${ }^{27-28}$

There are very few reports detailing the electropolymerization of CMP's that can be used for analyzing bioanalytes for biosensor applications. In 2015, that carbazole based microporous polymer networks (MPNs) deposited on a working electrode was reported by Palma-Cando et al. shows a response to nitro based explosive analytes. ${ }^{29}$ The obtained polymer film on a glassy carbon electrode (GC) was used as an electrochemical sensing platform to detect the nitroaromatic analyte 1,3,5-trinitrobenzene (TNB). The sensitivity of MPN to the analyte was achieved by the interaction between the electron deficient nitroaromatic analyte and the electron-rich MPN surface. ${ }^{30}$ The same group also studied the MPN's on various electrotrode surfaces to study the response to nitroaromatic analytes in comparison to plain GC. ${ }^{31}$ In 2018, Bai et al. electropolymerized 3,5-tri(thiophen-2-yl)benzene (TTB) on a GC electrode using chronoamperometry and cyclic voltammetric techniques to produce polymer films. They electropolymerized three monomers with a thiophene functional group on a GC electrode. The polymer films were then tested for the detection of metronidazole. The obtained results showed the elevation in the current response for the surface modified electrode with respect to the plain GC electrode. ${ }^{32}$ Recently, Hao et al. reported a multifunctional luminescent network polymer film, using triphenylethene groups with an anthracene core (TCzDPAn) which is an active blue emitting molecule for the detection of 2,4,6-trinitrophenol (TNP) in both vapour phase and solution using fluorescence resonance energy transfer (FRET) and photo-induced electron transfer (PET) techniques. The electropolymerised films were used as both a flourescent sensor as well as an OLED material. The electroluminescent device made using the electropolymerized film displayed a luminance of $923 \mathrm{~cd} / \mathrm{m}^{2}$ and $0.28 \mathrm{~cd} / \mathrm{A}$ of maximum luminance efficiency. This demonstrates the potential for future electroluminescent devices based on electropolymerized thin films. ${ }^{33}$ Ma et al. also reported poly $(3,4-$ 
ethylenedixythiophene) grafted with polyglycerol (PEDOT-HPG) using an electrochemical approach on a GC electrode. This was used to detect human cervical carcinoma cells via conjugation of alpha-fetaprotein (AFP) antibodies to the polymer film surface. The film showed a sensitivity level of $0.035 \mathrm{pg} / \mathrm{mL}$ with significant antifouling performance in complex biological media. $^{34}$

MicroRNAs have shown a great promise as disease specific biomarkers owing to their ubquitous regulatory role in almost all cellular processes. Moreover, as disease specific miRNAs are found in all bodily fluids either as free circulating molecules or enclosed in exosomes, they are particularly useful for development of minimally invasive liquid biopsy based disease diagnostic platforms. Molecular biology based miRNA detection methods such as RT-qPCR and next generation sequencing though are very sensitive, they are mostly expensive and not ideally suited for near-patient or resource limited settings. In recent years much attention has been paid to the development of electrochemical biosensors targeting various biomarkers including miRNAs for disease diagnostic applications. However, major drawback of most of these electrochemical biosensors is that they involve complicated sensor fabrication steps or in cases where redox reporters are used for signal amplification, loss of dynamic range is obesreved. Direct affinity interaction of biomolecules with electrode materials is one of the ways to circumvent these limitations of electrochemical biosensors and gold-DNA/RNA and graphen-DNA/RNA interaction has been exploited in a range of biosensing applications including miRNAs We have also previously demonstrated the potential applicability of thin films of positively charged poly(O-DPPN) electropolymerised on glassy carbon electrodes as miRNA sensing platform.

In this work, we have studied a new trifunctional conjugated monomer system of 1,3,5tri(furan-2-yl) benzene with three electron rich furan moieties attached at the 1, 3 and 5 positions of the central benzene ring. The trifunctional monomer was electropolymerized using chronoamperometry and cyclic voltammetry where a comparison between both methods is reported for its influence on the morphology of the electropolymerized films. Here, a threedimensional mesoporous conjugated network was employed on the flexible indium tin oxide (FITO) electrode. The obtained CMP's on FITO were further characterized using various techniques such as, X-ray photoelectron spectroscopy (XPS), scanning electron microscopy (SEM) and UV-vis spectroscopy and finally the conjugated CMP porous network was further utilized for detecting miRNA from the novel overian cancer cell.

\section{Experimental}




\subsection{Materials}

The rationally designed monomer 1,3,5-tri(furan-2-yl) benzene was purchased from VBR molecules, India. Silver with a purity of $99.9 \%$ and Platinum Wire (A\&E Metals, Australia) were used as reference electrodes and counter respectively. An flexible ITO (ITO deposited on a flexible polyethylene terephthalate (PET)) substrate with dimensions of $8 \mathrm{~mm} \times 20 \mathrm{~mm}$ was purchased from Yan Technology LTD Hong Kong, whereas aluminium tape was purchased from Techninstro ${ }^{\mathrm{TM}}$. An electrochemical cell with slots for the working electrode, counter and reference electrodes were used for this experiment. Ferrocene (Sigma Aldrich, Australia) was used as an internal standard for electrochemical experiments and all potentials are quoted to the $\mathrm{Fc} / \mathrm{Fc}^{+}$redox couple. Anhydrous dichloromethane (DCM) and Tetrabutylammonium phosphate (TBAP), are from Sigma Aldrich, Australia. The entire study was conducted on analytical grade and has been used directly for the experiments. Electropolymerization was conducted in the DCM medium with $0.1 \mathrm{M}$ TBAP as a supporting electrolyte. A twenty minutes purging under Argon was conducted before of every electrochemical experiment.

\subsection{Instrumentation and Characterization}

XPS measurements were done with a Kratos Axis ULTRA X-ray Photoelectron Spectrometer incorporating a $165 \mathrm{~mm}$ hemispherical electron energy analyser. The incident radiation was monochromatized Al Ka X-rays (1486.6 eV) at $150 \mathrm{~W}(15 \mathrm{kV}, 15 \mathrm{~mA})$. Survey (wide) scans were taken at an analyser pass energy of $160 \mathrm{eV}$ and high-resolution scans at $20 \mathrm{eV}$. Survey scans were carried out over a binding energy range from 1200 to $0 \mathrm{eV}$ with $1.0 \mathrm{eV}$ steps and a dwell time of $100 \mathrm{~ms}$. Narrow high-resolution scans were run with $0.05 \mathrm{eV}$ steps and $250 \mathrm{~ms}$ dwell time. Base pressure in the analysis chamber was $1.0 \times 10^{-9}$ Torr and during sample analysis was $1.0 \times 10^{-8}$ torr. Atomic concentrations were calculated using the CasaXPS version 2.3.14 software and a Shirley background with Kratos library Relative Sensitivity Factors (RSFs). Peak fitting of the high-resolution data was also carried out using the CasaXPS software. UV-Vis absorption spectra were recorded using a Cary 50 probe UV-Vis spectrometer.

SEM images were acquired with a Zeiss Sigma FE SEM, typically using accelerating voltages of $\sim 3.0 \mathrm{kV}$. Images were collected using both the secondary electron and the in-lens detectors, 
which have different contrast mechanisms that helped to elucidate image features. Results are presented here from only the secondary electron detector. Images were processed and analysed using ImageJ software. ${ }^{35}$ The false-colour in Figure 4 has been mapped to the polymer film. The corresponding unprocessed images are presented in the supporting information as Figures $\mathrm{S} 2$ and $\mathrm{S} 3$.

A four-point probe multimeter was used to measure the conductivity of the polymer films and the film thickness was measured by while a Brukers Dektak ${ }^{\circledR}$ XT Stylus Profiler instrument. The electrochemistry experiments were conducted using an Autolab PGSTAT204 potentiostat. A customised three-point electrode cell was in which the FITO was used as the working electrode. A DCM was used as a conducting medium with a supporting electrolyte of $0.1 \mathrm{M}$ TBAP and a monomer concentration of $5 \mathrm{mM}$.

All solutions for miRNA purification were prepared in UltraPure ${ }^{\mathrm{TM}}$ DNase/RNase-Free Distilled Water (Invitrogen). Known concentrations of synthetic miRNA of $22 \mathrm{bp}$ length were magnetically purified as described earlier with slight modifications. Briefly, target miRNA was hybridised to complementary capture probe (CP) by mixing $20 \mu \mathrm{L}$ of specific miRNA concentration with $30 \mu \mathrm{L}$ of $10 \mu \mathrm{M}$ biotinylated $\mathrm{CP}$ and $20 \mu \mathrm{L} 5 \mathrm{X}$ SSC buffer (pH 4) followed by one hour incubation at room temperature. $20 \mu \mathrm{L}$ of streptavidin-labeled Dynabeads MyOne $^{\mathrm{TM}}$ Streptavidin C1 (Invitrogen, Australia) were washed as per manufacturer's directions ( 2 times $1 \mathrm{x}$ binding and wash $(\mathrm{B} \& \mathrm{~W})$ buffer, 2 times with solution $\mathrm{A}(0.1 \mathrm{M} \mathrm{NaOH}$, $0.05 \mathrm{M} \mathrm{NaCl})$ for 2 minutes, and once with solution $\mathrm{B}(0.1 \mathrm{M} \mathrm{NaCl}))$ and resuspended in 70 $\mu \mathrm{L}$ of solution $\mathrm{B}$. Washed beads were subsequently mixed with target-probe mixture and incubated on a thermomixer for 30 mins at room temperature. Magnetic beads with attached miRNA targets were magnetically separated and washed twice before being finally resuspended in $20 \mu \mathrm{L} 5 \mathrm{x}$ SSC buffer. Beads were subsequently heated at $95^{\circ} \mathrm{C}$ for 5 minutes to release captured miRNA targets. Supernatant containing released miRNA was separated magnetically from the beads. Released target miRNAs were further diluted to $200 \mu \mathrm{L}$ total volume with $5 \mathrm{x}$ SSC.

A CHI650 electrochemical workstation ( $\mathrm{CH}$ instrument, USA) was used to carry out electrochemical sensing experiments. Immobilisation of miRNAs on the polymer surface was monitored as previously described with slight modifications. ${ }^{36} 50 \mu \mathrm{L}$ of purified miRNA was smeared onto the polymer coated electrodes and incubated at room temperature for 20 minutes. Differential pulse voltammetry (DPV) measurements were conducted in the presence of $50 \mu \mathrm{M}$ ruthenium hexaammine(III) chloride $\left(\left[\mathrm{Ru}\left(\mathrm{NH}_{3}\right)_{6}\right]^{3+}\right.$, RuHex) solution in $40 \mathrm{mM}$ Tris-buffer, 
before and after incubation with purified miRNA. Post-incubation with miRNA solution, electrodes were dipped in RuHex solution for 5 minutes prior to DPV measurements. DPV signals were recorded -0.7 to $0.05 \mathrm{~V}$ with a width of $50 \mathrm{mV}$ and a pulse amplitude of $50 \mathrm{mV}$. Each miRNA concentration was run in triplicate.

\section{Results and Discussion}

\subsection{Electropolymerization Studies}

There are several reports on CMP's with a thiophene moiety that form a three-dimensional conjugated polymer network when synthesised either chemically ${ }^{4,37}$ or electrochemically. ${ }^{31}$ However, there are a very few reports on electropolymerizing furan substituted monomers. Furan has a similar electropositive nature as thiophene and is also known as one of the most important biological degradable green conjugated building blocks. ${ }^{38}$ There are far fewer reports on furan based organic semiconductors in the literature and therefore this promising moiety was explored in this electropolymerization study.

We investigated the electropolymerization of a furan substituted trifunctional monomer namely, 1,3,5-tri(furan-2-yl) benzene (TFB), under various conditions to obtain conjugated polymer films on a FITO electrode. The electrochemical cell employed in this work is shown in Figure 1. Electropolymerization of TFB was investigated in a potential range of -1.5 to +2.0 $\mathrm{V}$ using cyclic voltammetry at a scan rate of $20 \mathrm{mV} / \mathrm{s}$. Ferrocene was used as an internal standard. The response for the TFB monomer at the FITO electrode shows a prominent oxidation peak and two reduction peaks at $1.00 \mathrm{~V}$ and 0.18 and $-0.25 \mathrm{~V}$ respectively (Figure 2A). The thoroughly washed electropolymerized film poly(TFB) was then characterised in background electrolyte only and exhibited a similar voltametric profile with an oxidation peak at $0.98 \mathrm{~V}$ and two reduction peaks at 0.72 and $0.04 \mathrm{~V}$, respectively (Figure 2B). Several films were made where the number of potential cycles were varied and consisted of 1,3 and 5 cycles. It is noticed that, the oxidation peak potential of the monomer is higher than its corresponding conjugated microporous polymers, as is the current passing via electropolymerized film is highly insulating than its corresponding monomer. ${ }^{39}$ The $1^{\text {st }}$ cycle of TFB on FITO for the monomer and subsequent polymer film are shown in Figure 2 while more cycles are shown in Figure S2. We also employed chronoamperometry to electropolymerize TFB on FITO to see 
if the method of electrodeposition would affect the electrochemical properties of the film. The working electrode potential was set to $+2 \mathrm{~V}$ and was applied for different time periods of 5,10 , 25, 50 and 75 seconds at a FITO electrode using $5 \mathrm{mM}$ TFB in $0.1 \mathrm{M} \mathrm{TBAPF} / \mathrm{DCM}$ (Figure 3). The sharp increase in current is due to capacitive charging followed by a gradual decrease in current due to formation of polymer on the surface of the electrode. The resultant poly(TFB) film fabricated using 5 seconds of deposition exhibits an oxidation peak and reduction peaks at $0.67 \mathrm{~V}$ and $0.04 \mathrm{~V}$. When the deposition time was increased to 10 seconds, an oxidative peak was seen at $0.73 \mathrm{~V}$ as well as a reduction peak at $-0.25 \mathrm{~V}$. Further increasing the time to 25 seconds resulted in two prominent oxidation peaks at $0.76 \mathrm{~V}$ and $1.62 \mathrm{~V}$ and a sharp reduction peak at $-0.11 \mathrm{~V}$. With an increase in deposition time to 50 seconds, the oxidation peak shifts slightly to $0.79 \mathrm{~V}$ and the reduction peak occurs at $0.12 \mathrm{~V}$. By increasing the time to 75 seconds very broad polymer oxidation and reduction peaks are observed at 0.93 and $0.03 \mathrm{~V}$ respectively. The electropolymerized films on FITO was confirmed by XPS analysis, SEM, UV-Vis.

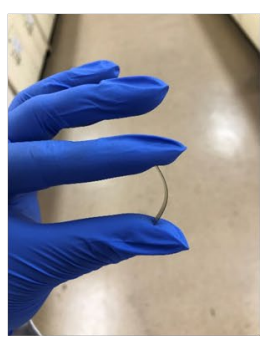

Substrate FITO

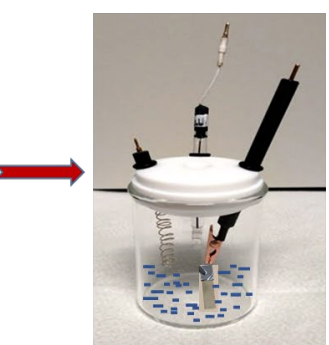

Electrochemical Cell Setup
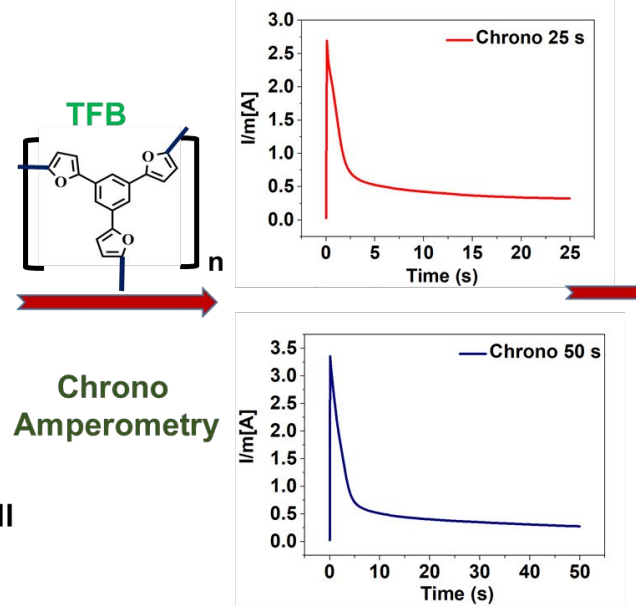

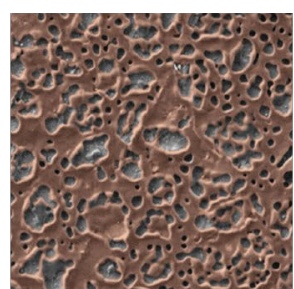

poly (TFB) on FITO

Figure 1: Electrochemical cell set up and chronoamperometry graphs for electropolymerization of poly(TFB) at $25 \mathrm{~s}$ and $50 \mathrm{~s}$ on FITO with the monomer concentration of $5 \mathrm{mmol} \mathrm{dm}^{-3}$ in dichloromethane containing 0.1 M TBAP as supporting electrolyte. 

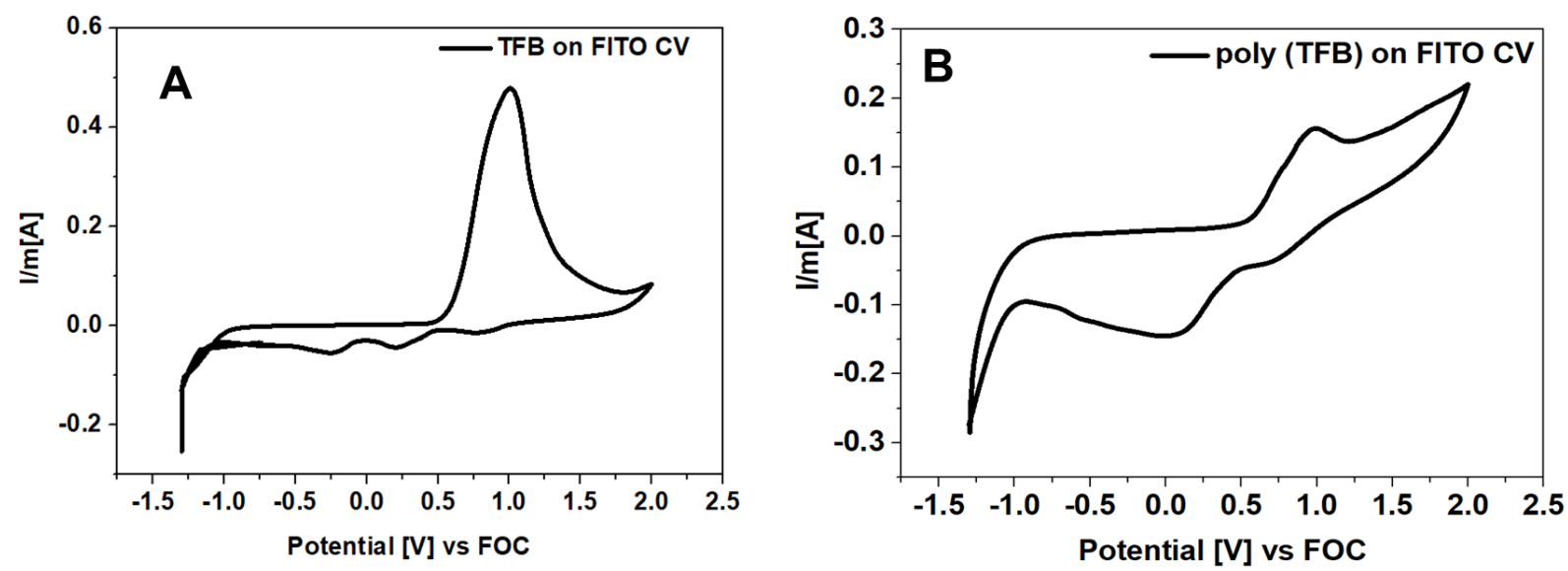

Figure 2: Cyclic voltammograms showing the $1^{\text {st }}$ cycle for the TFB monomer at a FITO electrode (A) and the response for poly(TFB) on FITO (B) in $0.1 \mathrm{M}$ TBAP in DCM as a supporting electrolyte containing a monomer concentration of $5 \mathrm{mmol} \mathrm{dm}^{-3}$, and a scan rate of $20 \mathrm{mV} / \mathrm{s}$.
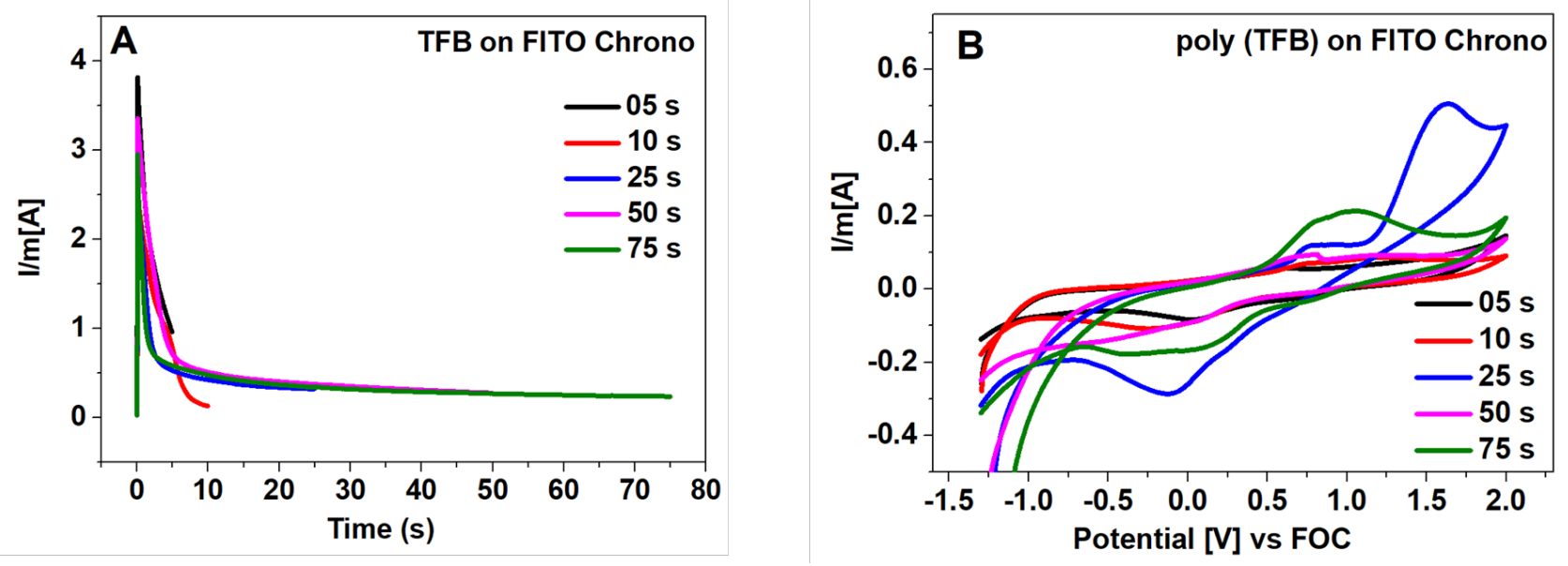

Figure 3: Chronoamperometry curves for the electropolymerization of TFB (A) held at $2.0 \mathrm{~V}$ for $5,10,25,50,75$ seconds and (B) corresponding cyclic voltammograms of poly(TFB) in in $0.1 \mathrm{M}$ TBAP in DCM as a supporting electrolyte containing a monomer concentration of 5 $\mathrm{mmol} \mathrm{dm} \mathrm{d}^{-3}$, and a scan rate of $20 \mathrm{mV} / \mathrm{s}$.

\subsection{Surface Morphology}

Scanning Electron Microscopy (SEM) Analysis 
The surface morphology of the electropolymerized films produced using chronoamperometry for time periods of 25 and 50 seconds was observed by SEM. Figure 4 depicts the false-colour SEM images of these films. The polymer film obtained after $25 \mathrm{~s}$ contains a high density of small pores with an average size $\sim 1.8 \mu \mathrm{m}^{2}$, whereas the polymer poly (TFB) film after $50 \mathrm{~s}$ exhibits fewer but larger pores, ca. $10-50 \mu \mathrm{m}^{2}$. The diameter of the pore size significantly changed with deposition time from 5 to $75 \mathrm{~s}$, as given in the Figure S3. The pore size is relatively small at 5 seconds, whereas at $75 \mathrm{~s}$ the electrode surface is completely covered with a polymer film. This correlates with the conductivity value of the polymer films which is obtained from four-point probe instrument, where the polymer layer at 5 seconds is semiconducting with a conductivity value of $1.84 \times 10^{3}$ Siemens $/ \mathrm{cm}$ whereas at $75 \mathrm{~s}$ the film was found to be insulating. Polymer films were also produced using a repetitive potential cycling approach over the potential range shown in Figure 2A. In these films, the pore size also increased in number and size with additional deposition cycles (Figure S4), however the pores were not as well as defined as seen for constant potential deposition (Figure 4).

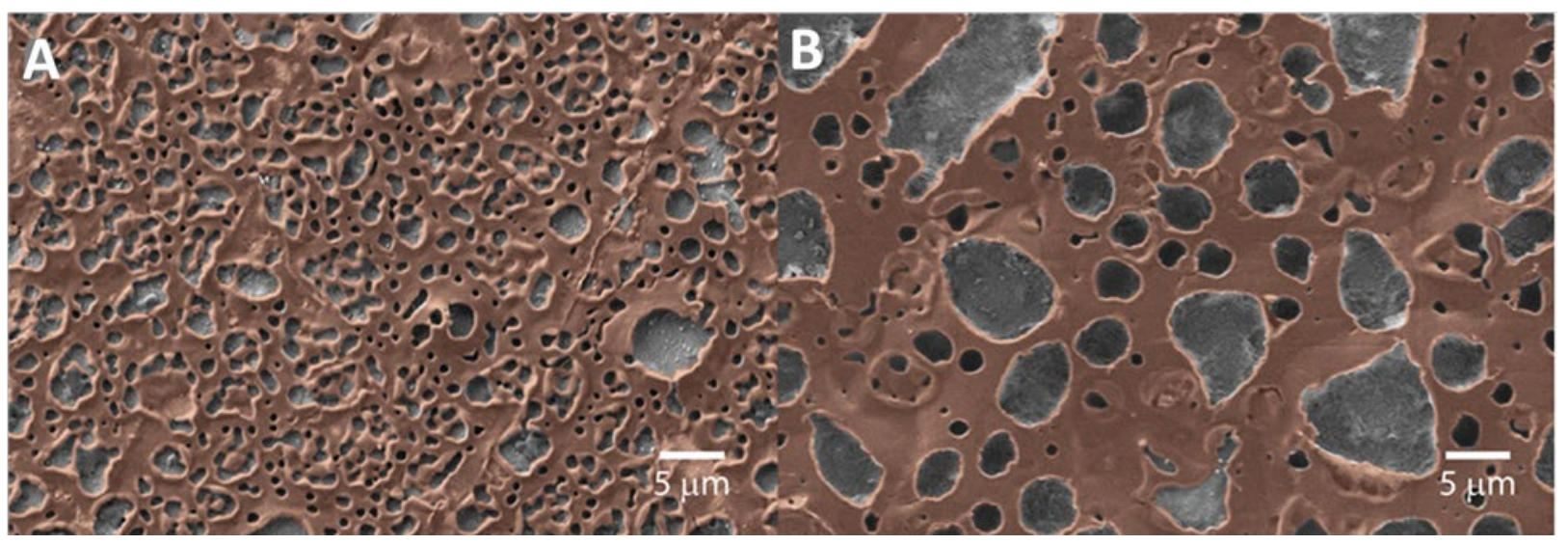

Figure 4: SEM images of poly (TFB) electropolymerized by chronoamperometry for 5 seconds (A) and 50 seconds (B) on a FITO electrode.

The surface thickness values of the poly(TFB) films on FITO for various deposition cycles and time are listed in Table S1 (see Supporting Information). The Supporting Information Table S2 provides the conductivity measurements of the polymer film. In our previous work, we electropolymerized a naphthalene flanked DPP monomer which clearly demonstrated how substrate conductivity and surface roughness of the electrode play an important role in polymer 
thin film forming properties. ${ }^{36}$ The surface roughness of FITO is $20 \mathrm{~nm}$ whereas in our previous work, glassy carbon (GC) and rigid ITO electrodes exhibited surface roughness values of ca. 40-50 $\mathrm{nm}$ and $1 \mathrm{~nm}$ respectively. Similarly, the conductivity of the substrates differs where FITO has a resistance of less than $6 \Omega$, whereas rigid ITO was reported to be less than $10 \Omega$, and GC is higher at $45 \Omega$. Thus, it can be inferred that FITO provides more sites for nucleation and growth with good conductivity and may serve as a better electrode when compared to rigid ITO and GC for number of nucleation sites and electrode conductivity, respectively. It was found that the poly(TFB) films formed on FITO at a constant potential for 25 and 50 seconds are stable and form three-dimensional structures which in principle may be highly suitable for biosensor applications. $^{2}$

\subsection{Optical Characterization of the Polymers}

A comparative study of the monomer and the electropolymerized polymer films was performed using UV-visible spectroscopy (Figure 5). The TFB monomer was spin coated onto a cleaned FITO substrate to generate a thin film which shows a relatively small absorption peak in the ultraviolet (UV) region from 300-450 nm . The absorption in this region may be associated with $\sigma$ bonds of the carbon rings of the monomer. There is a long tail extending from 450 to $700 \mathrm{~nm}$ indicating absorption corresponding to the $\pi$ to $\pi^{*}$ transition due to the double bond in the ring of the molecule. ${ }^{40}$ In UV -Vis region, the poly(TFB) film shows strong absorption as well as at higher wavelength in the visible region, indicating polymer formation. Poly(TFB) on FITO has two peaks: a strong peak at $350 \mathrm{~nm}$ indicating strong absorption from the $\sigma$ to $\sigma^{*}$ transition of the core repeating units of the polymer and a broad peak in the visible region due to $\pi$ to $\pi^{*}$ transitions between alternate units of the polymer. As the thickness of the polymer increases with deposition time from 5 to $75 \mathrm{~s}$, so does the relative absorption which clearly indicates the growth of multiple layers of the polymer as a function of time. 


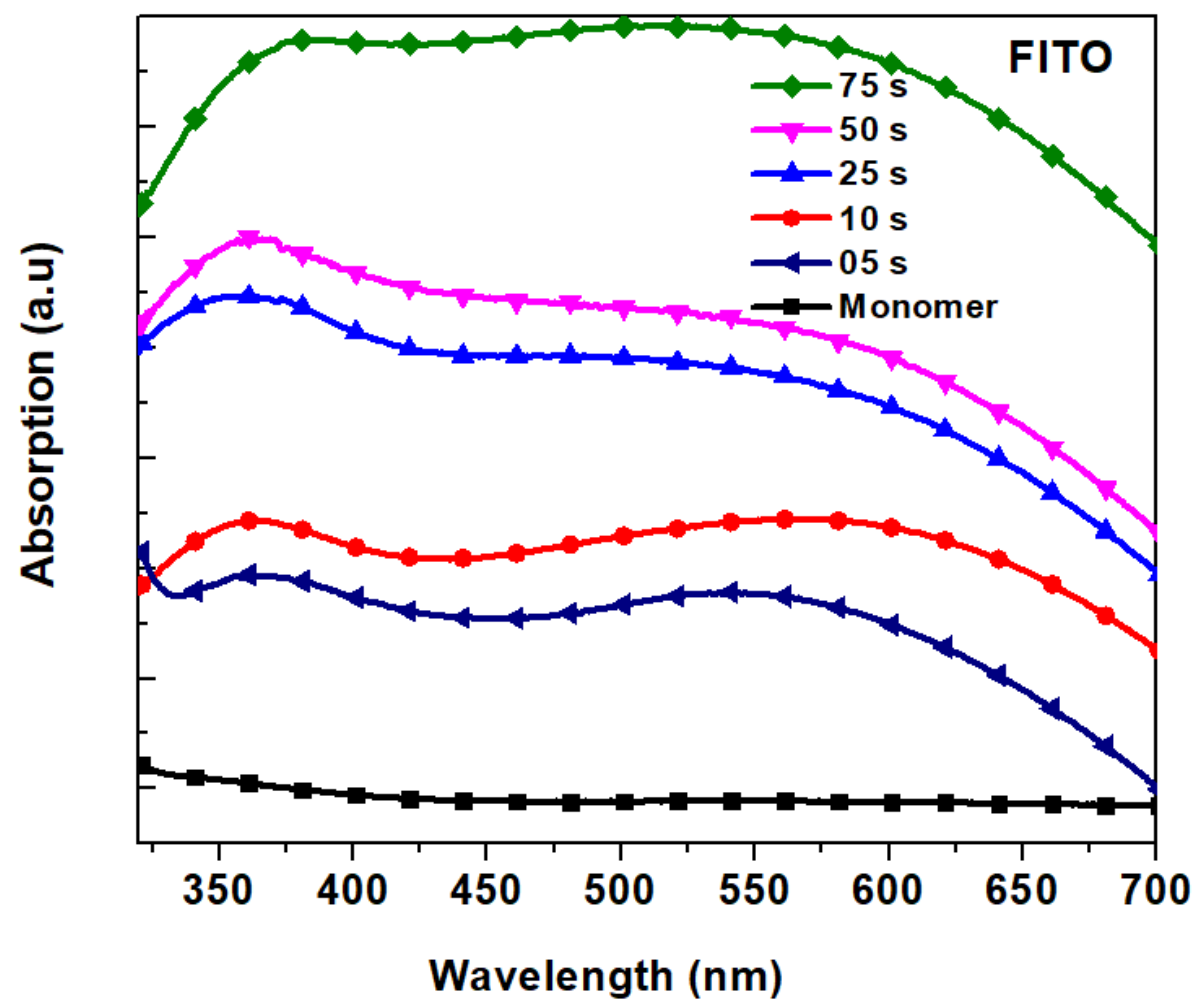

Figure 5: UV-Visible absorption spectra of poly(TBF) on FITO electropolymerized by chronoamperometry at various time intervals such as $5,10,25,50,75$ seconds in DCM containing 0.1 M TBAP as supporting electrolyte.

\subsection{X-ray Photoelectron Spectroscopy}

Figures $6 \mathrm{~A}, 6 \mathrm{~B}$ and $6 \mathrm{C}$ shows the $\mathrm{XP}$ spectra of the polymer film on FITO, the monomer on FITO and plain FITO. The XPS wide spectrum of the polymer did not show any peaks of indium and tin compared to the spin-coated monomer which substantiates the formation of a uniform layer of polymer on the substrate after electropolymerization. The XPS results clearly exhibit peaks of carbon and oxygen, which constitutes the elements of the polymer. For both polymers, the elemental composition measured by XPS is in acquiescence with the expected stoichiometries. Residual quantities of nitrogen and chlorine arising from the electrolyte used were detected, which is due to ions from the electrolyte trapped in the polymer backbone. The $\mathrm{C} 1 \mathrm{~s}$ spectrum can be deconvolved into three components, showing the presence of at least three 
types of carbon bonds that is, $\mathrm{sp}^{2}$ carbons in the conjugated core $(\sim 284.3 \mathrm{eV})$ and $\mathrm{sp}^{3}$ carbons in the substituents $(\sim 284.8 \mathrm{eV})$ and furan ring (C-O-C) and positively charged carbon at $(\sim 285.8 \mathrm{eV}) .{ }^{41-43}$ The difference in full width at half maxima (FWHM) of $\mathrm{sp}^{2}$ and $\mathrm{sp}^{3}$ deconvoluted peak indicates either that $\mathrm{sp}^{2}$ on the surface of polymer has reacted with oxygen and/or localised differential charging of the charge state on the surface of polymer thin film. The O1s spectrum can be deconvolved into two peaks with a major peak at $\sim 531.5 \mathrm{eV}$ corresponding to the furan ring (C-O-C) and a small peak at $\sim 530 \mathrm{eV}$ which can be attributed to the presence of metal oxides, in this case from the ITO substrate. The raster scan of XPS has dimensions in micrometre, so effect of substrate is evident, because of micro porous nature of thin film.
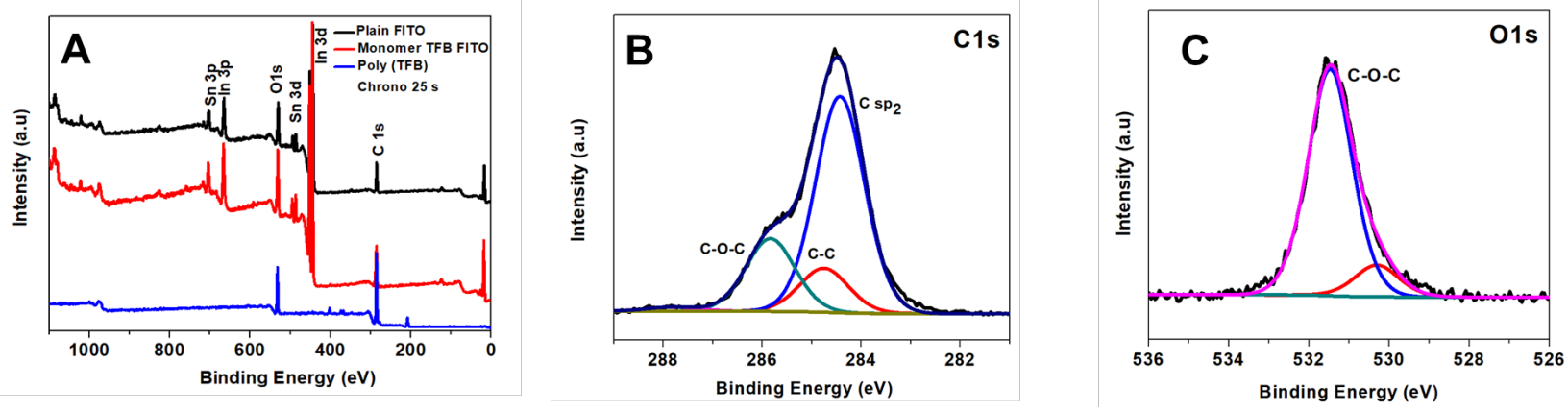

Figure 6: (A) XPS spectra of Plain FITO, (B) Monomer TFB on FITO and poly(TBF) on FITO and (C) poly(TFB) electropolymerized by chronoamperometry in the solution of $0.1 \mathrm{M}$ TBAP as supporting electrolyte in DCM .

\subsection{Application of the Electrochemically deposited poly(TFB) Thin Film}

We have previously demonstrated the application of novel electropolymerized thin films on glassy carbon electrodes to genosensing. ${ }^{36}$ The mesoporous nature of the poly(TFB) thin film electropolymerised on FITO may be a more sensitive genosensing platform due to the high surface area of this sensing layer. In order to test the applicability of the electropolymerized thin film on FITO, the sample produced by chronamperometry for $25 \mathrm{~s}$ (Figure 4A) was used due to its high stability and mesoporous structure. We challenged poly(TFB) coated FITO 
electrodes with clinically relevant concentrations of a novel ovarian cancer biomarker miRNA ( $1 \mathrm{nM}$ and $100 \mathrm{pM}$ starting concentrations). The known concentration of target miRNA was magnetically purified using biotinylated probe and streptavidin coated Dynabeads (Figure 7). Use of complemenatry probes specific for the target miRNA ensures the specificity of the assay. We employed the electrostatic interaction of cationic redox molecule, RuHex, to monitor the possible immobilization of miRNA on the polymer coated electrodes ${ }^{44-46}$. In addition to the polymer coated FITO electrodes, DPV measurements for unmodified FITO electrodes were also conducted. Figure 8 shows typical DPV measurements before and after deposition of miRNA on modified FITO electrodes as well as electrochemical response of RuHex on unmodified FITO electrodes. A substantial increase in the current was noticed after incubation with miRNA suggesting possible immobilization of miRNA on the polymer coated electrode surface which in turn leads to electrostatic accumulation of RuHex with the miRNA phosphate backbone which ultimately increased the current response. We tested two different starting concentrations of synthetic miRNA ( $1 \mathrm{nM}$ and $100 \mathrm{pM})$. Our data showed direct correlation between the change in current response and the miRNA concentration (Figure 8). Moreover, good reproducibility was observed between the replicates (RSD values $<5 \%$ ). As can be seen in Figure 8, slightly higher current response was obtained for unmodified FITOs which is in line with the conductivity/resitance measurements carried out for polymers modified and plain FITOs (Table S2). Although these preliminary results have demonstrated that this novel genosensing platform may be capable of detecting sample nucleic acids concentraions as low as $100 \mathrm{pM}$ (further diluted 1:10 post-purification), better control of electrode surface area as well as surface morphology of electropolymerized thin film may be needed to determine the dynamic range of the assay. 


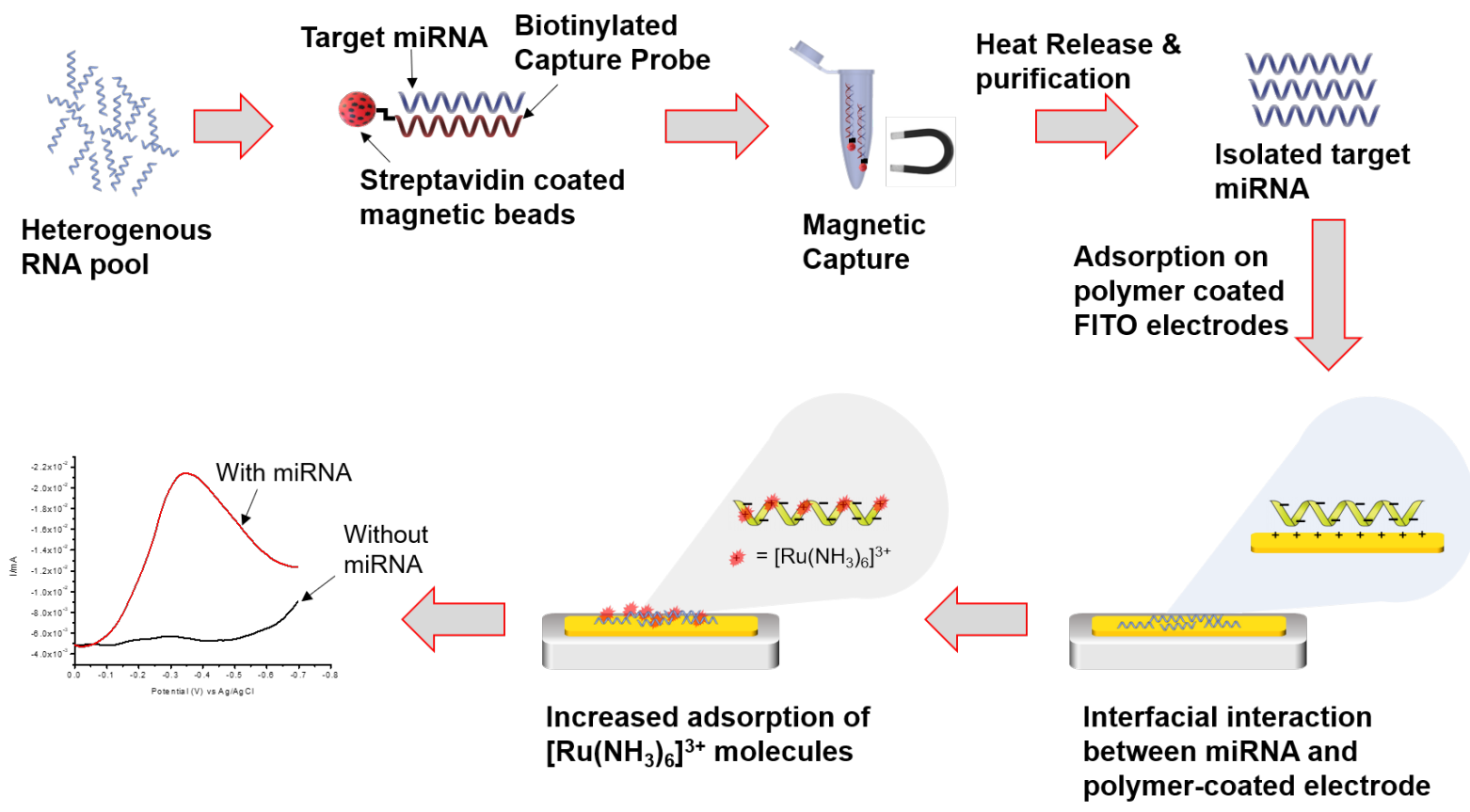

Figure 7: Schematic representation of biosensing assay. Traget miRNA was captured by using complementary biotinylated probes immobilized on Streptavidin-coated magnetic beads. The target miRNA were subsequently purified magnetically before being released from capture probes via heating. The isolated and purified targets were then directly adsobed onto a polymercoated FITO electrode. An increase in Faradaic current proportional to the concentration of miRNA was observed due to commensurate accumulation of $\left[\mathrm{Ru}\left(\mathrm{NH}_{3}\right)_{6}\right]^{3+}$ ions on to the electrode surface post-miRNA adsorption, thus enabling quantitative miRNA detection. 


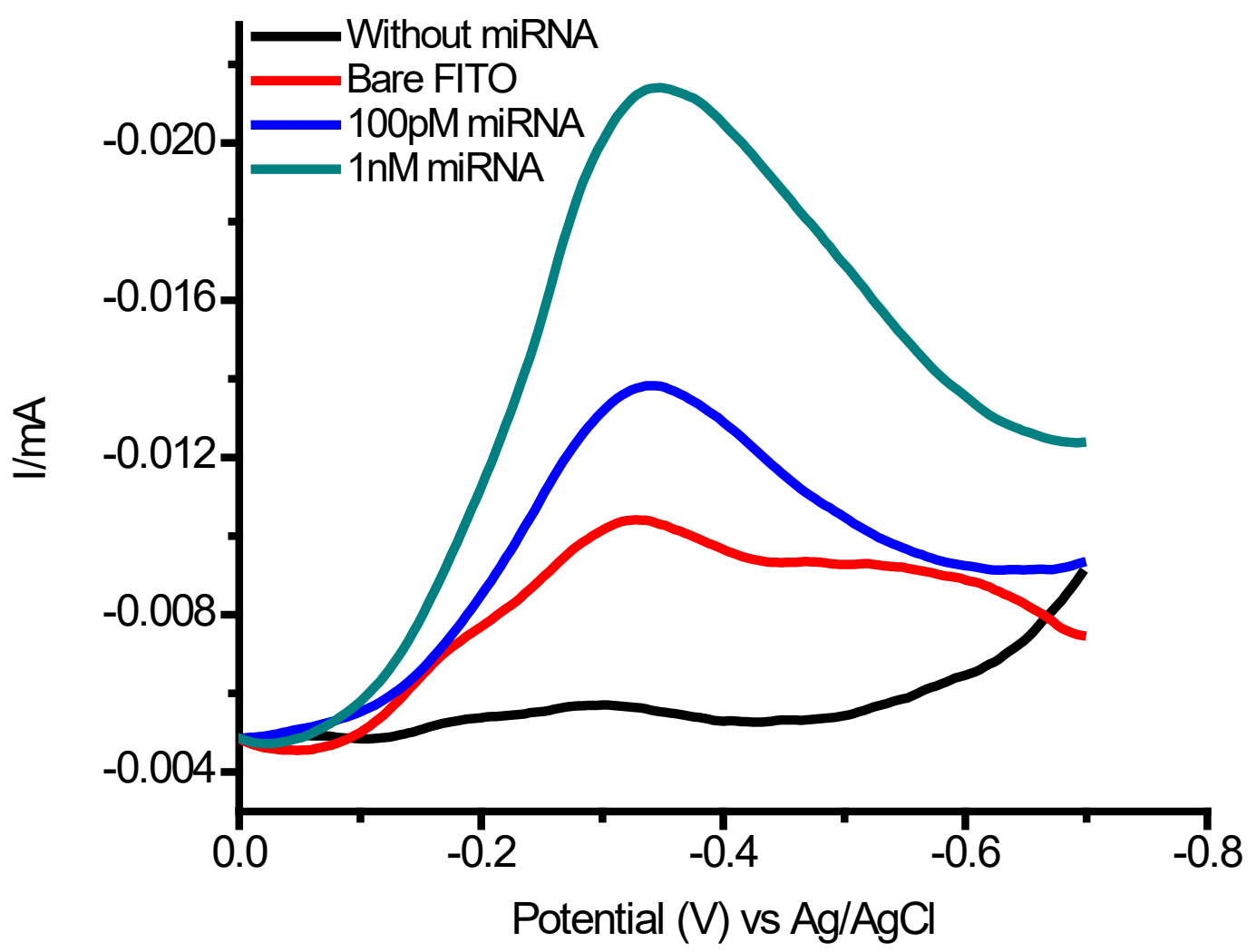

Figure 8: Effect of deposition of two purified miRNA concentrations on polymer coated FITO electrodes: DPV responses obtained before and after incubation with known miRNA concentrations.

\section{Conclusion}

A furan based conjugated microporous polymer poly (TFB) was synthesized using a one-step electrochemical method for the first time. The electropolymerized thin films achieved via constant potential deposition were further characterized by scanning electron microscopy, Xray photoelectron spectroscopy and UV-vis spectroscopy, confirming the formation of CMP films which showed a porous morphology whereby the pore size distribution depends on the deposition time. In addition, poly (TFB) films obtained by repetitive potential cycling did not exhibit a mesoporous structure indicating the importance of the electrochemical method used to produce these polymer films. The mesoporous poly (TFB) film on FITO proved to be a highly sensitive genosensing platform capable of detecting clinically relevant miRNA concentrations under point-of-testing related sample handling conditions. This approach could 
be applicable to other trifunctional monomers to produce conjugated porous polymers and could possibly be used for sensing applications.

\section{Acknowledgement}

Author is thankful to the CRC for Polymers and Central Analytical Research Facility (CARF), Institute of Future Environments, Queensland University of Technology (QUT) for providing equipment support. We are thankful to Centre for Microscopy and Microanalysis (CMM) University of Queensland (UQ) and XPS technician Barry Wood for the assistance in XPS. We are also thankful to Jickson Joseph from QUT for helping us in XPS analysis. P. S is thankful to QUT for the financial support from QUT core funding (QUT/ 322120-0301/07). P.S also acknowledges to the Australian Research Council (ARC) for the Future Fellowship (FT130101337).

\section{References}

(1) Das, T. K.; Prusty, S. Review on conducting polymers and their applications. Polymer Plast. Technol. Eng. 2012, 51 (14), 1487-1500.

(2) Gu, C.; Huang, N.; Gao, J.; Xu, F.; Xu, Y.; Jiang, D. Controlled Synthesis of Conjugated Microporous Polymer Films: Versatile Platforms for Highly Sensitive and Label-Free Chemoand Biosensing. Angew. Chem. Int. Ed. 2014, 53 (19), 4850-4855.

(3) Jiang, J. X.; Su, F.; Trewin, A.; Wood, C. D.; Campbell, N. L.; Niu, H.; Dickinson, C.; Ganin, A. Y.; Rosseinsky, M. J.; Khimyak, Y. Z. Conjugated microporous poly (aryleneethynylene) networks. Angew. Chem. Int. Ed. 2007, 46 (45), 8574-8578.

(4) Xu, Y.; Jin, S.; Xu, H.; Nagai, A.; Jiang, D. Conjugated microporous polymers: design, synthesis and application. Chem. Soc. Rev. 2013, 42 (20), 8012-8031.

(5) Gu, C.; Huang, N.; Chen, Y.; Zhang, H.; Zhang, S.; Li, F.; Ma, Y.; Jiang, D. Porous organic polymer films with tunable work functions and selective hole and electron flows for energy conversions. Angew. Chem. Int. Ed. 2016, 55 (9), 3049-3053.

(6) Wang, W.; Zhou, M.; Yuan, D. Carbon dioxide capture in amorphous porous organic polymers. J. Mater. Chem. A. 2017, 5 (4), 1334-1347.

(7) Zeng, Y.; Zou, R.; Zhao, Y. Covalent organic frameworks for $\mathrm{CO}_{2}$ capture. Adv. Mater. 2016, 28 (15), 2855-2873. 
(8) Liu, J.; Zou, R.; Zhao, Y. Recent developments in porous materials for $\mathrm{H}_{2}$ and $\mathrm{CH}_{4}$ storage. Tetrahedron Lett. 2016, 57 (44), 4873-4881.

(9) Bai, L.; Phua, S. Z. F.; Lim, W. Q.; Jana, A.; Luo, Z.; Tham, H. P.; Zhao, L.; Gao, Q.; Zhao, Y. Nanoscale covalent organic frameworks as smart carriers for drug delivery. Chem. Commun. 2016, 52 (22), 4128-4131.

(10) Mitra, S.; Sasmal, H. S.; Kundu, T.; Kandambeth, S.; Illath, K.; Díaz Díaz, D.; Banerjee, R. Targeted drug delivery in covalent organic nanosheets (CONs) via sequential postsynthetic modification. J. Am. Chem. Soc. 2017, 139 (12), 4513-4520.

(11) Su, C.; Tandiana, R.; Tian, B.; Sengupta, A.; Tang, W.; Su, J.; Loh, K. P. Visible-light photocatalysis of aerobic oxidation reactions using carbazolic conjugated microporous polymers. Acs Catalysis. 2016, 6 (6), 3594-3599.

(12) Jia, H.; Sun, Z.; Jiang, D.; Du, P. Covalent cobalt porphyrin framework on multiwalled carbon nanotubes for efficient water oxidation at low overpotential. Chem. Mater. 2015, 27 (13), 4586-4593.

(13) Wu, K.; Guo, J.; Wang, C. An elastic monolithic catalyst: A microporous metalloporphyrin-containing framework-wrapped melamine foam for process-intensified acyl transfer. Angew. Chem. Int. Ed. 2016, 55 (20), 6013-6017.

(14) Li, Z.; Li, H.; Xia, H.; Ding, X.; Luo, X.; Liu, X.; Mu, Y. Triarylboron-linked conjugated microporous polymers: sensing and removal of fluoride ions. Chem. Eur. J. 2015, 21 (48), $17355-17362$.

(15) Sang, N.; Zhan, C.; Cao, D. Highly sensitive and selective detection of 2, 4, 6trinitrophenol using covalent-organic polymer luminescent probes. J. Mater. Chem. A. 2015, 3 (1), 92-96.

(16) Bandyopadhyay, S.; Pallavi, P.; Anil, A. G.; Patra, A. Fabrication of porous organic polymers in the form of powder, soluble in organic solvents and nanoparticles: a unique platform for gas adsorption and efficient chemosensing. Polym. Chem. 2015, 6 (20), 37753780.

(17) Jiang, J.-X.; Laybourn, A.; Clowes, R.; Khimyak, Y. Z.; Bacsa, J.; Higgins, S. J.; Adams, D. J.; Cooper, A. I. High surface area contorted conjugated microporous polymers based on spiro-bipropylenedioxythiophene. Macromolecules. 2010, 43 (18), 7577-7582.

(18) Bao, Z.; Chan, W.; Yu, L. Synthesis of conjugated polymer by the stille coupling reaction. Chem. Mater. 1993, 5 (1), 2-3. 
(19) Qian, X.; Zhu, Z.-Q.; Sun, H.-X.; Ren, F.; Mu, P.; Liang, W.; Chen, L.; Li, A. Capture and reversible storage of volatile iodine by novel conjugated microporous polymers containing thiophene units. ACS Appl. Mater. Interfaces. 2016, 8 (32), 21063-21069.

(20) Liras, M.; Iglesias, M.; Sánchez, F. 1. Conjugated microporous polymers incorporating BODIPY moieties as light-emitting materials and recyclable visible-light photocatalysts. Macromolecules. 2016, 49 (5), 1666-1673.

(21) Yuan, K.; Guo-Wang, P.; Hu, T.; Shi, L.; Zeng, R.; Forster, M.; Pichler, T.; Chen, Y.; Scherf, U. Nanofibrous and graphene-templated conjugated microporous polymer materials for flexible chemosensors and supercapacitors. Chem. Mater. 2015, 27 (21), 7403-7411.

(22) Schmidt, J.; Werner, M.; Thomas, A. Conjugated microporous polymer networks via yamamoto polymerization. Macromolecules. 2009, 42 (13), 4426-4429.

(23) Chen, Q.; Liu, D. P.; Luo, M.; Feng, L. J.; Zhao, Y. C.; Han, B. H. Nitrogen-containing microporous conjugated polymers via carbazole-based oxidative coupling polymerization: preparation, porosity, and gas uptake. Small. 2014, 10 (2), 308-315.

(24) Kobayashi, N.; Kijima, M. Microporous materials derived from two-and threedimensional hyperbranched conjugated polymers by thermal elimination of substituents. $J$. Mater. Chem. 2007, 17 (40), 4289-4296.

(25) Sonar, P.; Foong, T. R. B.; Dodabalapur, A. Synthesis of diketopyrrolopyrrole based copolymers via the direct arylation method for p-channel and ambipolar OFETs. Phys. Chem. Chem. Phys. 2014, 16 (9), 4275-4283.

(26) Szczepanski, C. R.; M'Jid, I.; Darmanin, T.; Godeau, G.; Guittard, F. A template-free approach to nanotube-decorated polymer surfaces using 3,4-phenylenedioxythiophene (PhEDOT) monomers. J. Mater. Chem. A. 2016, 4 (44), 17308-17323.

(27) Gu, C.; Chen, Y.; Zhang, Z.; Xue, S.; Sun, S.; Zhang, K.; Zhong, C.; Zhang, H.; Pan, Y.; Lv, Y. Electrochemical route to fabricate film-like conjugated microporous polymers and application for organic electronics. Adv. Mater. 2013, 25 (25), 3443-3448.

(28) Gu, C.; Huang, N.; Wu, Y.; Xu, H.; Jiang, D. Design of highly photofunctional porous polymer films with controlled thickness and prominent microporosity. Angew. Chem. Int. Ed. 2015, 54 (39), 11540-11544.

(29) Palma-Cando, A.; Woitassek, D.; Brunklaus, G.; Scherf, U. Luminescent tetraphenylethene-cored, carbazole-and thiophene-based microporous polymer films for the chemosensing of nitroaromatic analytes. Mater. Chem. Front. 2017, 1 (6), 1118-1124. 
(30) Palma-Cando, A.; Scherf, U. Electrogenerated thin films of microporous polymer networks with remarkably increased electrochemical response to nitroaromatic analytes. $A C S$ Appl. Mater. Interfaces. 2015, 7 (21), 11127-11133.

(31) Palma-Cando, A.; Brunklaus, G.; Scherf, U. Thiophene-based microporous polymer networks via chemical or electrochemical oxidative coupling. Macromolecules. 2015, 48 (19), 6816-6824.

(32) Bai, S.; Hu, Q.; Zeng, Q.; Wang, M.; Wang, L. Variations in surface morphologies, properties, and electrochemical responses to nitro-analyte by controlled electropolymerization of thiophene derivatives. ACS Appl. Mater. Interfaces 2018, 10 (13), 11319-11327.

(33) Hao, H.; Luo, H.; Yi, A.; Liu, C.; Xu, B.; Shi, G.; Chi, Z. A multifunctional luminescent network film electrochemically deposited from a new AIEE emitter for OLEDs and explosive detection. Org. Electron. 2019, 69(1), 281-288.

(34) Ma, L.; Jayachandran, S.; Li, Z.; Song, Z.; Wang, W.; Luo, X. Antifouling and conducting PEDOT derivative grafted with polyglycerol for highly sensitive electrochemical protein detection in complex biological media. J. Electroanal. Chem. 2019, 840, 272-278.

(35) Chouhan, R.; Chouhan, M.; Shrivastava, B.; Mishra, A.; Phadke, S. Microscopic analysis of natural and reinforce bar of phoenix sylvestris. Int. Jour. Inno. Know. Conc. 2019, 7 (2), 9497.

(36) Ponnappa, S.; Umer, M.; Liu, Q. N.; MacLeod, J.; Jickson, J.; Ayoko, G. A.; Shiddiky, M. J.; O'Mullane, A. P.; Sonar, P. M. Naphthalene flanked diketopyrrolopyrrole: A new conjugated building block with hexyl or octyl alkyl side chains for electropolymerization studies and its biosensor applications. Polym. Chem. 2019,10, 3667-3798.

(37) Qin, L.; Xu, G.-j.; Yao, C.; Xu, Y.-h. Thiophene-based conjugated microporous polymers: preparation, porosity, exceptional carbon dioxide absorption and selectivity. Polym. Chem. 2016, 7 (28), 4599-4602.

(38) Gu, C.; Huang, N.; Chen, Y.; Qin, L.; Xu, H.; Zhang, S.; Li, F.; Ma, Y.; Jiang, D. $\pi$ Conjugated microporous polymer films: designed synthesis, conducting properties, and photoenergy conversions. Angew. Chem. Int. Ed. 2015, 54 (46), 13594-13598.

(39) Calderon, E. H.; Dangate, M.; Manfredi, N.; Abbotto, A.; Salamone, M. M.; Ruffo, R.; Mari, C. M. Electrochemical and spectroelectrochemical properties of a new donor-acceptor polymer containing 3, 4-dialkoxythiophene and 2, 1, 3-benzothiadiazole units. Polymers. 2013, 5 (3), 1068-1080. 
(40) Winokur, M.; Spiegel, D.; Kim, Y.; Hotta, S.; Heeger, A. Structural and absorption studies of the thermochromic transition in poly (3-hexylthiophene). Synth. Met. 1989, 28 (1-2), 419426.

(41) Gora, M.; Krzywiec, W.; Mieczkowski, J.; Rodrigues Maia, E. C.; Louarn, G.; Zagorska, M.; Pron, A. Alternating copolymers of diketopyrrolopyrrole or benzothiadiazole and alkoxysubstituted oligothiophenes: spectroscopic, electrochemical and spectroelectrochemical investigations. Electrochim. Acta. 2014, 144, 211-220,

(42) Glenis, S.; Benz, M.; LeGoff, E.; Schindler, J.; Kannewurf, C.; Kanatzidis, M. Polyfuran: a new synthetic approach and electronic properties. J. Am. Chem. Soc. 1993, 115 (26), 1251912525.

(43) Del Valle, M.; Ugalde, L.; Díaz, F.; Bodini, M.; Bernède, J. Effect of working conditions on the morphology of electrosynthesized polyfuran. J. Appl. Polym. Sci. 2004, 92 (2), 13461354.

(44) Sina, A. A. I.; Carrascosa, L. G.; Palanisamy, R.; Rauf, S.; Shiddiky, M. J.; Trau, M. Methylsorb: a simple method for quantifying DNA methylation using DNA-gold affinity interactions. Anal. Chem. 2014, 86 (20), 10179-10185.

(45) Koo, K. M.; Carrascosa, L. G.; Shiddiky, M. J.; Trau, M. Amplification-free detection of gene fusions in prostate cancer urinary samples using mrna-gold affinity interactions. Anal. Chem. 2016, 88 (13), 6781-6788.

(46) Masud, M. K.; Islam, M. N.; Haque, M. H.; Tanaka, S.; Gopalan, V.; Alici, G.; Nguyen, N.-T.; Lam, A. K.; Hossain, M. S. A.; Yamauchi, Y. Gold-loaded nanoporous superparamagnetic nanocubes for catalytic signal amplification in detecting miRNA. Chem. Commun. 2017, 53 (58), 8231-8234. 
